\title{
Nível de informação da população e utilização dos mecanismos institucionais de participação social em saúde em dois municipios do Nordeste do Brasil
}

\author{
Level of community information and use \\ of mechanisms for social participation in health \\ in two municipalities of Northeast Brazil
}

María Luisa Vázquez 1,2

Maria Rejane Ferrei ra da Silva 2,3

Eliane Siqueira Campos Gonzalez 4

Alcides da Silva Diniz 5

Ana Paula Campos Pereira 4

Ida Cristina Leite Veras 4

Ilma Kruze Grande de Arruda 4,5

${ }^{1}$ ConsorciHospitalari

de Catalunya.

Av. Tibidabo 21, 08022,

Barcelona, Espanha.

mlvazquez@chc.es

2 Ins ti tute for Health Sector

Development, Londres.

3 Faculdade de Enfermagem

Nossa Senhora das Graças,

Un iversidade de

Pernambuco.

4 Instituto Ma terno

Infantil de Pernambuco.

5 Un iversid ade Federal

de Pernambuco.
Abstract A fundamental plank of the health sector reforms in Brazil is the democratization of health services by exercising social control upon the health system. A series of institutional mechanisms for social participation in the health services were designed. This paper analyses people's knowl ed ge and experien ces with institu tional direct mechanisms (Municipal Health Council, Health Conferences, Disque Saúde and health ombudsman) and an indire ct mechanism (PROCON). A questionnaire survey to a sample of 1590 health servi ces users was carried out, in two municipalities of Pernambuco. Around half of the interviewed population declared themselves aware of the existence of Disque Saúde, suggestions boxes and the Municipal Health Council. The other direct mechanisms were less well known and the PROCON was known by the majority of the intervi ewees (80\%). The functions of any mechanism, except for PROCON, were of ten inaccurately and vaguely defined. The take-up ra te did not exce ed 5\%. These re sults se em to indicate that some progress was made, but the challenge of tra n sfo rming legal gains into policy practices continues to remain, beginning with improving the provision of information to the population.

Key words Social participation in health policies, Participation mechanisms, Information levels, Utilization of participation mechanisms, Brazil, Latin America
Resumo A reforma do setor saúde no Brasil contempla como eixo fundamental a democratização dos serviços de saúde através do exercício do controle so cial sob re o sistema de saúde. Foram desenhados diversos mecanismos de participação nos serviços de saúde. No artigo analisam-se o nível de informação e a utilização pela população dos mecanismos de participação em saúde diretos: Con selhos Municipais de Saúde, Conferências de Saúde, Disque-Saúde e Ouvidoria de Saúde; e um indireto, a Superintendência de Proteção e Defesa do Con sumidor (Procon). Realizou-se um inquéri to populacional, com questionário estruturado, em uma amostra de 1.590 usuários dos serviços de saúde, em dois municípios de Pernambuco. Cerca de metade da população entrevistada afirmava conhecer o Disque Saúde, a Caixa de Queixas e o CMS; os outros mecanismos diretos eram muito menos conhecidos. A maioria dos entrevistados afirmou conhecer o Procon (80\%). A finalidade do mecanismo, exceto para o Procon, foi definida de forma va ga ou inexata. A taxa de utilização não superou $5 \%$. Os resultados parecem indicar que houve avanço, embora o desafío continue sendo levar à prática as conquistas no plano legal, começando por melhorar a informação à população.

Pal avras-chave Políticas de participação social em saúde, Mecanismos de pa rticipação, Níveis de informação, Utilização de mecanismos de participação, Brasil, América Latina 


\section{Introdução}

As políticas que prom ovem a participação social em saúde evolu íram ao lon go do tem po de acordo com as própias evoluções experim en tadas nas con cepções da saúde, os seus determinantes e o sistema de saúde. Embora tenham sido regis tradas experiências isoladas de participação da população em diversos projetos e programas de saúde pública desde finais do século 19, a participação comunitária aparece como questão cen tral nas políticas de saúde desde os anos 70. É um dos principios da estratégia da Atenção Primária de Saúde (OMS, 1978), in sere-se no locus da filosofía da promoção de saúde (OMS, 1986; 1988; 1989; 1995) e é uma das políticas introduzidas nas reformas dos sistemas de saúde no contexto da descentralizaç ã o e democra tização dos serviços de saúde (OMS 1996; Araújo, 1997; Cel edón \& Noé, 2000).

Diversos autores qu e s ti onam a participação social prom ovida pelas instituições e analisam as dificuldades de caráter político, cultural e econômico que essa participação en f renta (Valla, 1998a; Zakus \& Lysack, 1998; Dias, 1998a; Stone, 1992; Woelk, 1992). Como qualquer outra política pública, para sua implantação, devem con correr elemen tos políti cos - redistribuição de poder e recursos -, elementos or ganizativos e elementos relativos à população (Vázquez et al., 2002a). En tre os el em en tos organizativos, a serem de s envo lvi dos pelas instu ições, necessários para uma possível participação da sociedade nos serviços de saúde, se encontra a existência de "portas de entrad a" ao sistema ou mecanismos institucionais por meio dos quais os cidadãos possam incorporar a sua opinião e atividade, de manei ra col etiva ou individual, aos serviços de saúde, tais como os conselhos municipais ou sistemas de queixas e sugestões (González, 1996; Zakus \& Lysack, 1998). Muitas das políticas de participação social introduzidas no con tex to das reformas dos sis temas de saúde con templam a criação destes mecanismos (Bennett, 1998). Outro elemento básicopara a participação da população no sistema de saúde é o acesso à informação, como instrum en to fundamental para a democratização dos serviços (Kl ein, 1984; Zakus \& Lysack, 1998; Dias, 1998b). A população deve con h ecer os seus direitos em saúde, os problemas e as suas causas, as possíveis intervenções, os serviços oferecidos e sua qualidade, assim como os mecanismos pelos quais pode interagir com o sistema. A informação (das instituições à po- pulação sobre os projetos ou programas) é considerada o nível mínimo de participação nas escalas que analisam o grau de participação da população (González, 1996; Ars tein, 1969).

No Brasil, a participação da população é um dos ei xos principais na estrutu ração do Si stema Único de Saúde (SUS) e está claramente definida dentro do marco legal da reforma do s etor saúde (Constituição Federal, 1988; Leis 8.080 e 8.142, 1990; NOB's 1/91, 1/92 e 1/96). A Constituição Federal estabel ece que é um direi to e um dever de todo cidadão participar em todos os níveis de governo. E s te novo marco legal recolhe uma concepção democrática da p a rticipação em saúde, significando a integração, em parceria com o Estado, dos diferen tes setores da população na definição de políticas de saúde a serem implementadas, bem como no monitoramen to de sua implem en tação, incluindo aspectos econômicos e financeiros.

Os tex tos legais con templam a criação de diversos mecanismos institu cionais de participação para que a população possa exercer um con trole sobre o sistema de saúde. Além de revigorizar e criar mecanismos diretos de participação coletiva (Conselho de Saúde, Conferências de Saúde), prom ove o estabelecimen to de mecanismos para a participação individual: disque-saúde, caixas de queixas e sugestões e o uvidoria de saúde (GTCOM/Abra s co 2002). Os co nselhos de saúde são órgãos col egiados, de caráter permanen te e del ibera tivo, constituídos em todas as esferas de govern o, com participação paritá riados usuários, cu jas finalidades são formular estratégias para operacionalização das políticas setoriais e controlar a execução das políticas e ações de saúde, inclusive nos seus aspectos econô mi cos e financei ros. Iniciadas em 1941, e revigorizadas a partir de 1986, as Conferências de saúde se con stitu em em fóruns de repre sentação dos vários segm en tos sociais, para avaliar a situação de saúde e propor as diretrizes para a formulação da política de saúde nos níveis de governo correspondentes. São convocadas a cada quatro anos e sempre com a participação paritária do usuário em relação ao conjunto dos demais segmentos. O disque-saúdese con s ti tui em uma linha tel efônica para prestar informações e receber denúncias relativas à saúde. As ouvidorias de saúde têm como finalidade receber as reclamações de qualquer cidadão, as denúncias de quaisquer violações de direitos individuais ou coletivos relativos à saúde, bem como qualquer ato de improbidade administrativa, praticados por 
servidores públ i cos, a p u rar a veracid ade dos fatos e provi denciar as medidas cabívei s .

Do mesmo modo, se contempla a divulgação de informações sobre os meios legais existen tes para exi gir o cumprim en to das leis (Ministério Públ i co, Superintendência de proteção e defesa do consumidor (Procon), Conselhos Profissionais) e os meios de comunicação de massa, como mecanismos indiretos de participação em saúde (GTCOM/Abrasco).

Apesar destes avanços, alguns estu dos e relató rios oficiais regis tram a limitada participação da população, evidenciando o descompasso en tre as con quistas obtidas no plano formal e a prática cotidiana dos serviços de saúde (Dias, 1998a; Cohn, 2000; Cotta et al. 2000; Ministério da Saúde, 2002a; Valla, 1998b). Para a análise da implem entação de políticas em saúde, se devem levar em conta tanto elementos do con texto, con te údo e processo, como relativos aos própios atores sociais envo lvi dos (Walt \& Gilson, 1994). Embora diversos estudos analisem os fatores que influ enciam os processos de participação social em saúde, pa recem ser escassos os estu dos destinados a avaliar os níveis de informação da população sobre os mecanismos institucionais de participação, como condição prévia para sua possívd utilização, assim como a ex tensão da utilização destes mecanismos pela população em nível local. A maioria das publicações en con trad as, em relação com o processo brasileiro, se con cen tra, fundamentalmente, na dinâmica dos conselhos de saúde (Pe sso to et al., 2001; Abrantes-Pêgo, 1999; Wendhausen \& Caponi 2002; Pedrosa, 1997). Além disto, são ra ras as pes quisas sobre participação social em saúde (Mosquera et al., 2001) que realizem estudos de base populacional.

À luz dessa revisão, desenhou-se uma pesquisa destin ada a analisar diversos aspectos da implementação das políticas de participação social nos serviços de saúde, no con texto das a tuais reformas do setor saúde noBrasil. As diferen tes con cepções sobre participação social em saúde, dos agen tes principais e suas implicações para a implem entação das políticas, fora m analisadas e apresentadas em outros lugares (V á z quez et al. 2002b; 2003). Ne s teartigo se dis$c$ utem os resultados referen tes a um dos obj etivos da pesquisa, defin i do como avaliar o conhecimento e ex periências dos usu á rios com os mecanismos institucionais para a participação social nos serviços de saúde nas localidades estudadas. As análises proc u ram iden tificar a ex tensão da informação na população sobre seis dos mecanismos institucionais de participação social, assim como a frequência de sua utilização.

\section{Metodologia}

O desenho da pesquisa correspon de a um estudo descritivo transversal mediante inquérito domiciliar, no qual se procu rou dimen si onar o nível de informação dos usuários dos serviços de saúde sobre os mecanismos institucionais de participação em saúde e de sua utilização. Foram selecionados cinco mecanismos diretos, e um mecanismo indireto.

\section{Á rea de estudo}

O estudo foi realizado nos municípios do Cabo de Sa n to Agostinho, de caráter predom inantemente rural, e Camaragibe, de caráter predominantemente urbano, integrantes da Região Metropolitana de Recife, Estado de Pernambu co, no Nordes te brasilei ro. Os dois municípios foram selecionados de acordo com os seguintes critérios: nível máximo de descen tralização do sistema de saúde; den sidades demográficas contrastantes; implementação de reformas no setor saúde que inclu em a implantação de mecanismos institu cionais de participação social em saúde.

\section{Método}

Realiza-se um inquérito populacional, no perí odo en tre março e junho de 2000 . Utilizouse um question á rio con ten do perguntas abertas e fech adas sobre cinco mecanismos institu cionais de participação direta nos serviços de saúde, Con selho Municipal de Sa ú de (CMS), Conferências de Saúde, caixa de queixas e sugestões, disque-saúde, o uvi doria de saúde, e um indireto, o Procon. Foram incluídas perguntas sobre o conhecimento da existência, utilização, finalidade e funcionamen to dos mecanismos. Igualmente, foram incluídas perguntas sobre os mecanismos existentes nas unidades de saúde habitualmente utilizadas pelos en trevistados.

\section{Amostra estudada}

Calculou-se o tamanho da amostra com base na taxa de utilização dos mecanismos institucionais de participação nos serviços de saúde, estimado segundo os resultados obtidos do estu do piloto. Para uma população de 149.964 
habitantes no Ca bo e 118.968 em Camaragibe, estimou-se em 797 e 795, respectivamente, o número de indiví duos a serem incluídos no inqué ri to, admitindo-se um nível de confiança de 95\%, uma precisão de 3\% e uma taxa gl obal de utilização de mecanismos de participação insti tu cionais na ordem de $25 \%$. A amostra foi selecionada de forma aleatória, mediante uma s eleção sistem á tica dos domicílios. Calculou-se o intervalo amostral e sorteou-se o domicílio inicial do inquérito em cada setor censitário (IBGE) de domicílios. Finalmente, pa ra garantir uma boa repres entação masculina, se fez uma amostragem por cota em cada bairro. Foram en trevistadas 1.590 pessoas, 801 no Ca bo e 789 em Camaragi be (Ta bela 1).

\section{Qualidade dos dados e processamen to da informação}

O questionário foi testado em dois estudos piloto antes de assumir a forma definitiva. Durante a realização do inquéri to, todos os questionários eram revisados, procedendo-se à repetição de $20 \%$ das entrevistas (cerca de 318 ). Utilizou-se a técnica de dupla entrada para a digitação dos dados no programa Epi-info-6.0, em um banco provi do de mecanismos de controle para verificação automática de incon gruências. Rel a tó rios de freqüências simples a poi aram as análises de consistência. As respostas às perguntas abertas foram agrupadas por critério de similitude de con te ú do e, po s teri ormente, codificadas. Foram realizadas análises univariada e bivariada utilizando-se o programa SPSSWIN, versão 8.0. As proporções foram com paradas utilizando-se o te s te do $\mathrm{x}^{2}$ de Pearson, sendo adotado o nível de significância de 5\% para rej eição da hipótese denulid ade.

\section{Resultados}

\section{Características da população estudada}

Am bos os municípios apre sentam características semelhantes em relação à com posição dem ográfica, ní veis educacionais, ocupação e utilização de planos de saúde.

A proporção de mulh eres entrevistadas foi su perior à de homens, na cidade do Cabo $(\mathrm{p}=$ $0,001)$, embora semelhante à dos homens no município de Camaragi be $(\mathrm{p}=0,05)$ (Tabela 1$)$. Consideran do a população estudada como um todo, o percen tual en con trado de mulh eres foi de 54,2 (51,7-61,9); e de homens foi de 45,8 (95\% IC 43,4-48.3).

Apesar de em ambos os municípios as mulh eres apre sen ta rem um melhor nível de escolaridade em todos os ciclos de estu do em relação aos hom ens, apenas em Camaragi be ob servou-se que esta diferença era estatisticamente significativa $\left(x^{2}=17,74 ; p<0,001\right)$. A taxa de analfabetismo entre as mulheres do município do Cabo foi de $18,6 \%$ e a dos homens foi de 19,6\%. Em Camaragibe estas taxas foram respectivamente de $11,4 \%$ e de $17,4 \%$. As taxas de analfabetismo foram $19,1 \%$ e $14,2 \%$, respectivamente, no Cabo e em Camaragibe. Em todos os grupos de idade havia pessoas analfabetas. Aproximadamente $60 \%$ dos entrevistados dos dois municípios apresenta ram nível de escolaridade, no máximo, até o ciclo fundamental completo.

Quanto à ocupação, a proporção geral de trabalhadores manuais não qualificados ou parcialmen te qualificados foi de 49,9\% no Cabo e $52,7 \%$ em Camaragibe. Vale ressaltar que a proporção de mulh eres que realizavam ativi-

Tabela 1

Distri buição da amostra estudada por idade e sexo. Ca bo de Santo Ago s tinho e Camaragibe, 2000.

\begin{tabular}{|c|c|c|c|c|c|c|c|c|c|c|}
\hline \multirow[t]{3}{*}{ Idade } & \multicolumn{5}{|c|}{ Ca bo de Santo Agostinho } & \multicolumn{5}{|c|}{ Camaragibe } \\
\hline & \multicolumn{2}{|c|}{ Mulheres } & \multicolumn{2}{|c|}{ Homens } & \multirow[t]{2}{*}{ Total } & \multicolumn{2}{|c|}{ Mulheres } & \multicolumn{2}{|c|}{ Homens } & \multirow[t]{2}{*}{ Total } \\
\hline & $\mathrm{N}$ & $\%$ & $\mathrm{n}$ & $\%$ & & $\mathrm{n}$ & $\%$ & $\mathrm{n}$ & $\%$ & \\
\hline $13-19$ & 44 & 10,0 & 39 & 10,8 & 83 & 28 & 6,7 & 42 & 11,4 & 70 \\
\hline $20-30$ & 115 & 26,1 & 73 & 20,2 & 188 & 122 & 29,9 & 76 & 20,7 & 198 \\
\hline $31-45$ & 139 & 31,6 & 77 & 21,3 & 216 & 132 & 31,4 & 76 & 20,7 & 208 \\
\hline $46-65$ & 99 & 22,5 & 110 & 30,5 & 209 & 103 & 24,5 & 108 & 29,3 & 211 \\
\hline $66 e+$ & 43 & 9,8 & 62 & 17,2 & 105 & 36 & 8,6 & 66 & 17,9 & 102 \\
\hline \multirow[t]{2}{*}{ Total } & 440 & 54,9 & 361 & 45,1 & 801 & 421 & 53,4 & 368 & 46,6 & 789 \\
\hline & \multicolumn{5}{|c|}{ média $=41,8 ; \mathrm{DE}=18,3$} & \multicolumn{5}{|c|}{ média $=41,9 ; \mathrm{DE}=18,0$} \\
\hline
\end{tabular}


dades manuais não qualificadas foi de $66,7 \%$ no Ca bo e de 70,7\% em Camaragibe. A taxa de desemprego foi de, aproximadamente, 10,5\% nos dois municípios. Os apos en tados e pensionistas representavam, aproximadamente, 17,3\% da amostra do Cabo e 14,9\% da de Camaragibe, observan do-se ainda, uma menor proporção de mulheres aposentadas ou pensionistas (3,0\% no Ca bo e $2,0 \%$ em Camaragibe), quando comparada àquela observada entre os homens $(14,3 \%$ no Cabo $(\mathrm{p}<0,001)$ e $12,9 \%$ em Camaragi be $(\mathrm{p}<0,001)$.

Cerca do $15 \%$ dos en trevistados $(15,8 \%$ no Cabo e 15,0\% em Camaragibe) possuíam planos de saúde. Destes, $46,5 \%$ no Cabo e 51,3\% em Camaragibe, pagavam planos de saúde com cobertura individual ou familiar, sen do que $31,5 \%$ no Cabo e $41,2 \%$ em Camaragibe possuíam cobertu ra por vínculo com em presa privada ou pública. Dos restantes, $22 \%$ no Cabo e 7,6\% em Camaragibe, pagavam planos de saúde com uma limitada cobertura de assistência, como, por exemplo, plano odon tológi co ou de a tenção à gestante.

\section{Nível de informação e utilização dos mecanismos institucionais de participação em saúde}

No inquérito, realizou-se um conjun to de perguntas para dimensionar o nível de infor- mação, bem como a utilização pelos usuários dos mecanismos institu ci onais de participação, quais sejam: CMS, as Con ferências de Saúde, as Caixas de queixas e sugestões, o Disque-saúde, as Ouvidorias de Saúde e o Procon.

Perg u n tou-se a todos os en trevistados se sabiam da existência de cada um dos mecanismos supramencionados. O disque-saúde e o Procon foram os mecanismos mais conhecidos, seguidos pelo CMS; enquan to os menos conheci dos foram as Con ferências e a Ouvi doria de Saúde (Figura 1). À exceção da caixa de qu eixas e sugestões, todos os mecanismos eram mais conhecidos pelos entrevistados de Camaragibe, se bem que somen te foram identificadas diferenças estatisticamen te significativas en tre as proporções dos que con h eciam o Conselho de Saúde $\left(\chi^{2}=14,13 ; \mathrm{p}<0,001\right)$ e o Pro$\operatorname{con}\left(\chi^{2}=3,74 ; p<0,05\right)$ (Figura 1). Deve-se ainda de st acar que, em Camaragibe, a proporção de pessoas que con h eciam o CMS su perou àqu ela que conhecia a caixa de queixas e sugestões.

Questionou-se ainda se os entrevistados haviam utilizado esses mecanismos institu cionais de participação. As taxas de utilização não su peraram $5 \%$ para nen hum dos mecanismos. As maiores taxas, similares em ambas as localidades, foram as de utilização do Procon $(4,0 \%)$, a caixa de queixas e sugestões $(4,2 \%)$ e o disque saúde para os usuários de Camaragi be (Figura 2).

\section{Figura 1}

Proporção de entrevis tados que afirmaram con h ecer cada um dos mecanismos institu cionais de participação. Ca bo de Santo Ago s tinho e Camaragibe, 2000.

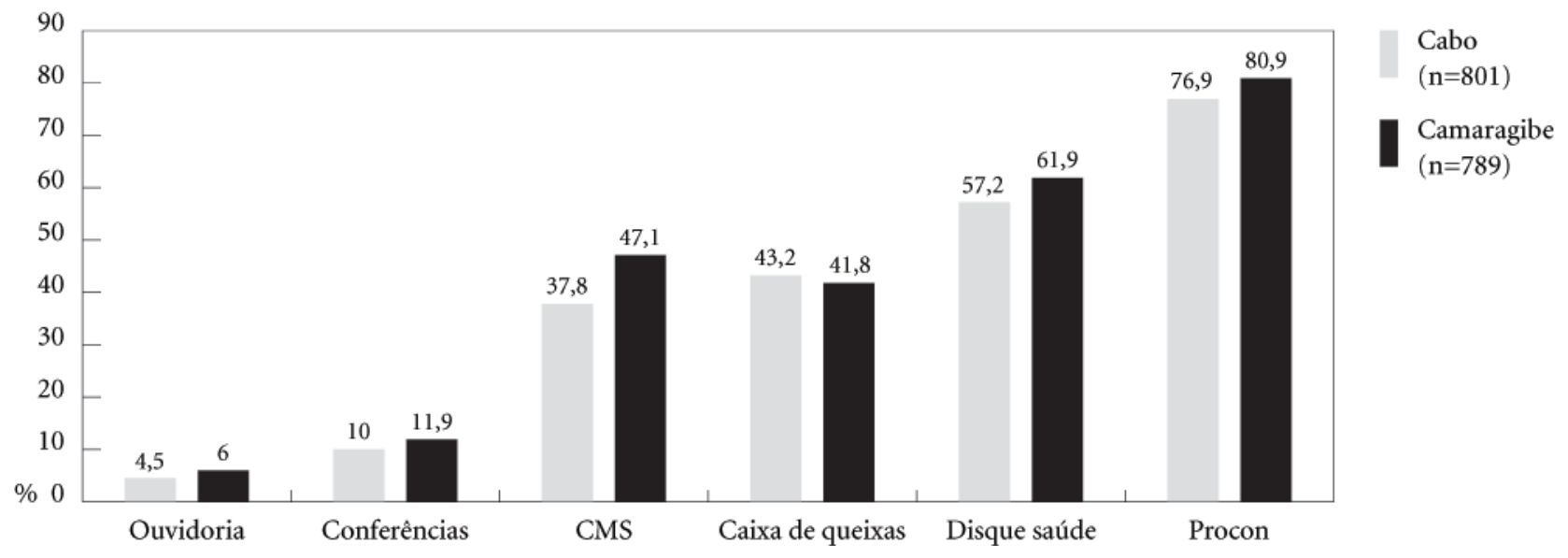


Taxa de utilização de cada um dos mecanismos institu ci onais de participação.

Ca bo de Santo Ago s tinho e Camaragibe, 2000.

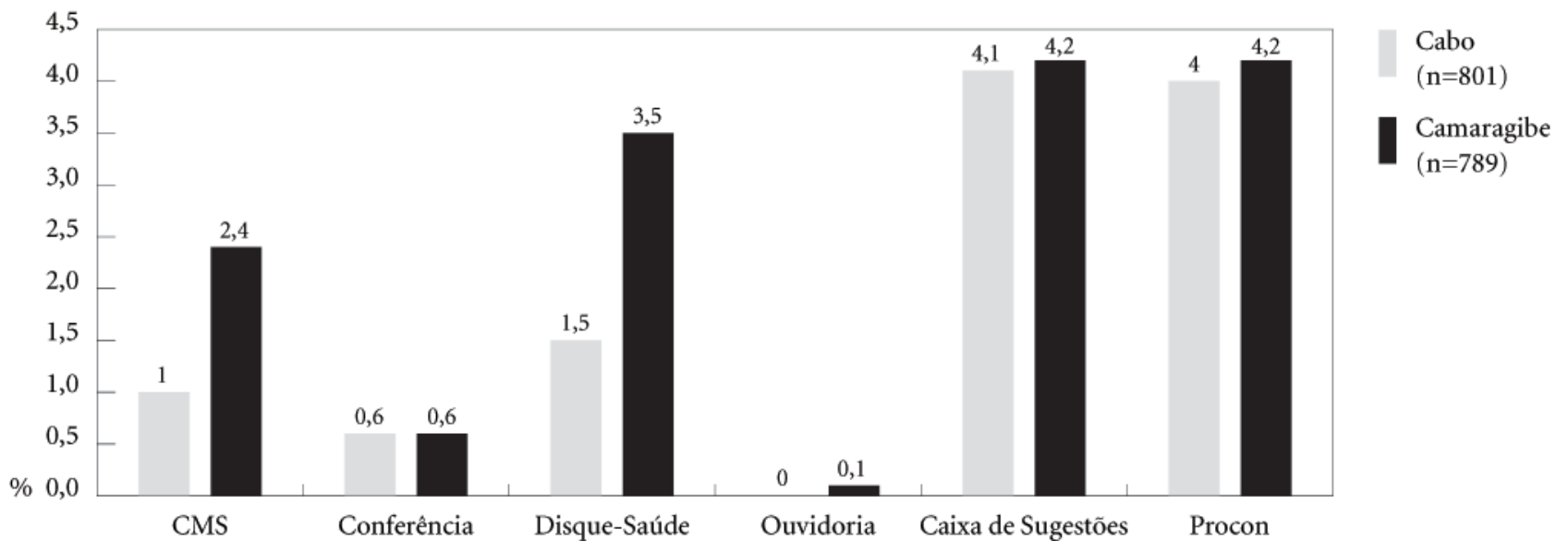

As perguntas complementares que permitiram aprofundar sobre o nível de conhecim ento e a experiência do entrevistado, com cada um dos mecanismos de participação, foram feitas àqueles que afirmaram conhecer a sua existência.

\section{Con selho Municipal de Saúde (CMS)}

Dos en trevistados que afirm a ram conhecer o CMS, cerca de $37,8 \%$ eram do Cabo de $47,1 \%$ de Camaragibe (Figura 1). Quandose perguntou para que servia este col egiado, as respostas foram pontuais e variadas; apenas $4,6 \% \mathrm{da}$ amostra, de ambas as localidades, re s pon deram de forma mais pertinente, se bem que as demais respostas tiveram um certo grau de acerto. Em torno de $16 \%$ das res postas eram de caráter genéri co, como por exemplo, "melhorar os serviços de saúde" (Figura 3). Uma importante proporção de en trevistados, ta n to no Cabo $(60,4 \%)$ como em Camaragibe $(50,4 \%)$, de sconhecia a finalidade do CMS.

Apenas 3,2\% dos en trevistados no Cabo e 9\% em Camaragibe afirmaram que conheciam seu representanteno CMS. Ainda assim, fora $\mathrm{m}$ muito poucos os entrevistados que identificaram, com su posta coerência, o seu repre s en t a nte no CMS. Quanto à difusão das atuações do CMS, os resultados revelam uma limitação nas vias e no alcance de informações. Apenas 3,5\% dos en trevist ados no Ca bo e 7,8\% em Camaragi be receberam informações sobre a atuação do CMS. Em ambos os municípios, os entrevistados disseram que recebiam informações por panfletos, folhetos, mídia e, particularmen te em Camaragibe, a metade deles, pelo jornal do município.

As experiências dos entrevistados com o CMS foram inexpressivas. Cerca de 1,0\% do Ca bo e 2,4\% de Camaragibe assistiram a alguma das reuniões do CMS (Figura 2). Do mesmo modo, m enos de $1 \%$ dos entrevistados do Cabo e 3,5\% dos de Camaragibe participaram em el eições para a escolha de conselheiros municipais de saúde. Nas duas localidades, a maioria participou das reuniões do CMS para denunciar probl emas que en con travam nos serviços de saúde e, em men or proporção, para se informar sobre a situação de saúde domunicípio.

\section{Con ferências de Saúde}

Os resultados do inquérito populacional revelaram que $10 \%$ da amostra do Ca bo e $12 \%$ da de Camaragibe conheciam as conferências de saúde (Figura 1). Es tes en trevistados, quando questionados sobre a finalidade destes encontros, apresentaram uma variedade de respostas gerais, alusivas ao debate em busca de soluções para os problemas dos serviços e do sistema de saúde, como também re s postas refe- 


\section{Figura 3}

Distri buição percentual das re spostas rela tivas à finalidade do Cons elho Municipal de Saúde.

Ca bo de Santo Ago stinho e Camaragibe, 2000.



ren tes ao intercâmbio de informações em saúde. Aproximadamen te $25 \%$ des tes en trevistados, nos dois municípios, desconheciam a finalidade das con ferências (Figura 4). Nas duas localidades, a penas cerca de $0,6 \%$ disse que havia participado de alguma conferência de saúde, nos últimos 5 anos (Figura 2).

\section{Caixas de qu eixas e sugestões}

No Ca bo, 43,2\% afirm a ram con h ecer a caixa de queixas e sugestões enqua n to em Camaragibe esta proporção foi de 41,7\% (Figura 1). Indagou-se a estes en trevistados qual era a sua opinião quan to ao encaminhamento dado pelos serviços de saúde às informações coletadas por este mecanismo. Em torno de 53,5\% dos entrevistados de Camaragibe e 43,5\% dos do Ca bo opinaram que as unidades de saúde analisavam, respondiam e procuravam melhorar s eus serviços com base nestas informações. Ao contrário, $42,5 \%$ dos entrevistados do Cabo e $33,7 \%$ dos de Camaragi be não ac reditavam que as demandas apresentadas recebes semalguma atenção por parte dos serviços (Figura 5).

No que se refere às experiências com a caixa de queixasesugestões, detectou-se que a taxa de utilização, em ambososmunicípios, foi de aproximadamen te $4 \%$ (Figura 2). As razões que levaram estes en trevist adbs a utilizá-la foram similares em ambas as localidades, tan to em proporção, como em con teú do. Aproximadamen te 30\% utilizaram para reclamar sobre o atendimen to ( qu eixas do médico, falta de fichas, etc), enquanto quase $70 \%$ dos entrevistados se motivaram para el ogiar ou dar sugestões sobre os serviços.

\section{Disque-saúde}

Destacou-se a expressiva proporção de indivíduos que afirmava con hecer o disque-saúde, representando 57,2\% dos en trevistados do Cabo e 61,9\% dos de Camaragibe (Figura 1). Foi questionado aos entrevistados para que servia este mecanismo; em ambos os municípios, em torno de $22 \%$ descreveram que este era um 
Figura 4

Distri buição percentual das res postas rel a tivas à finalidade das Con ferências de Saúde.

Ca bo de Santo Ago s tinho e Camaragibe, 2000.

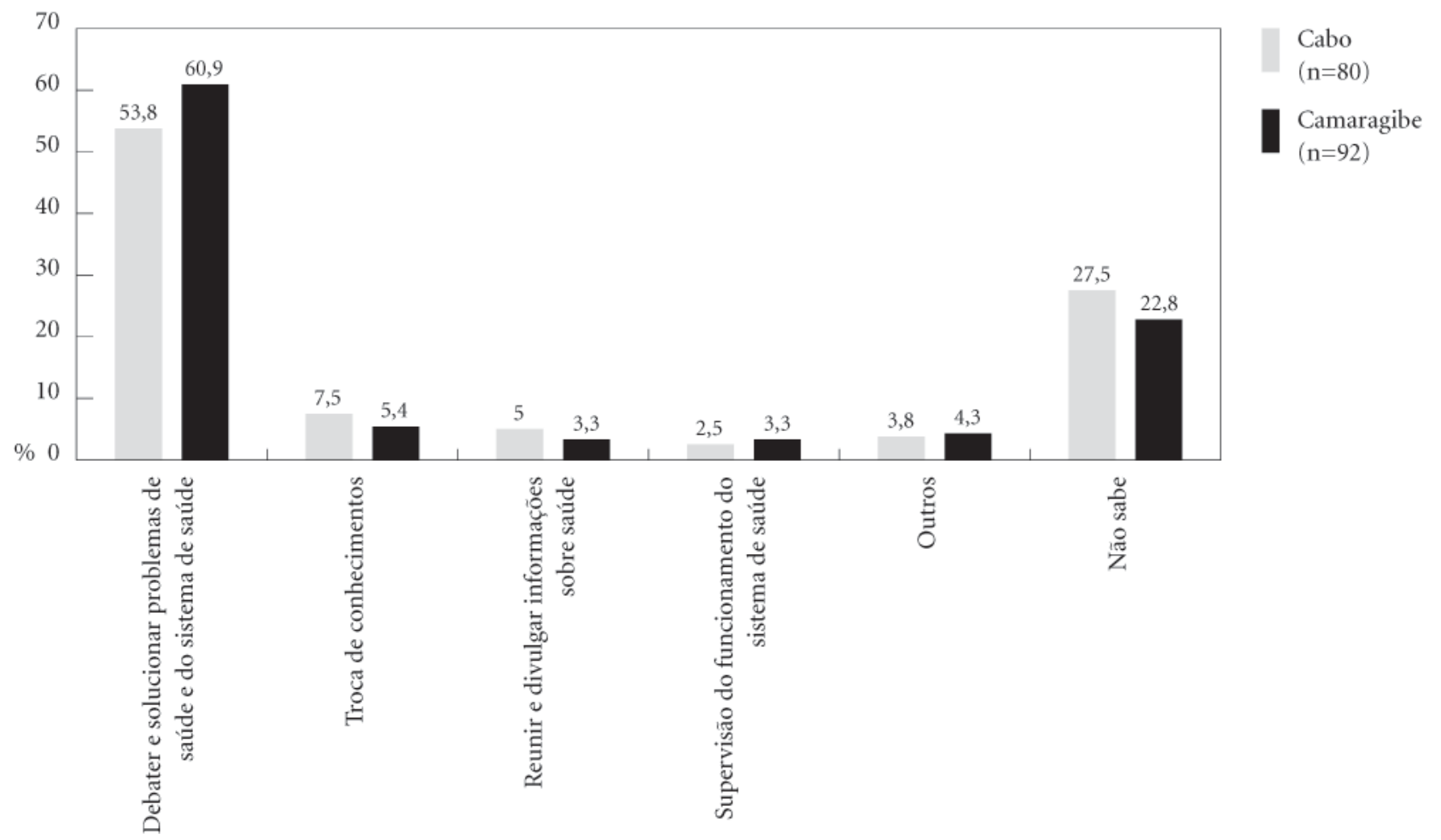

Figura 5

Distri buição percentual das re s postas sobre os procedim en tos realizados nos serviços com as queixas e sugestões.

Ca bo de Santo Ago stinho e Camaragibe, 2000.

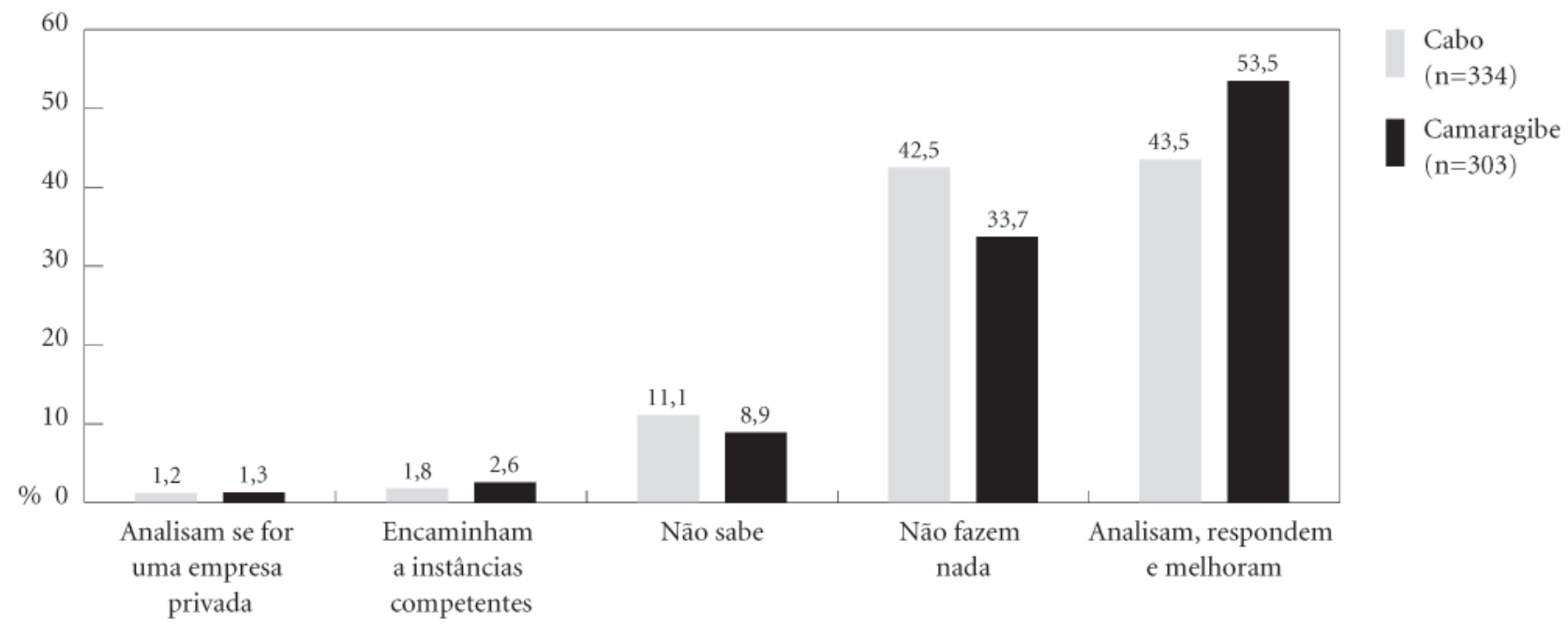


m ecanismo de participação destin ado a veicular informações e queixas. Uma boa proporção dos entrevistados, tan to no Cabo (39\%), como em Camaragibe (50\%), respondeu que o disque-saúde era um meio para facilitar o aten dimen to, com o, por exemplo, "m a rcar con sultas" ou "chamar a ambulância". A finalidade deste mecanismo não foi identificada por $38 \%$ dos entrevistados do Ca bo e por 25,7\% em Camaragibe (Figura 6). As experiências com o disque-saúde foram inexpre s sivas. No Cabo, a penas 1,5\% dos en trevistados realizou alguma ligação e em Camaragibe 3,5\% (Figura 2).

\section{O uvidoria de Saúde}

De todos os mecanismos de participação estudados, a Ouvi doria de Saúde era o menos conhecido e foi lembrado por apenas $4,5 \%$ da amostra do Cabo e por 6\% da de Camaragibe (Figura 1). Dos que sabiam da existência da O uvidoria de Saúde, a maioria não sabia qual era a sua finalidade ( $81 \%$ no Cabo e $59 \%$ em Camaragibe). Os demais entrevistados re sponderam que a Ouvidoria servia para escutar e resolver probl emas de saúde e do sistema de saúde, como também re solver probl emas do atendimen to e receber denúncias de abusos. Quanto às ex periências com este mecanismo, apenas uma pessoa de Camaragibe afirmou que o havia utilizado nos últimos 5 anos (Figura 2).

\section{Procon}

Den tre todos os mecanismos institucionais estudados, o Procon era o mais con hecido e foi lembrado por $76,9 \%$ da amostra no Ca bo e por $80,9 \%$ em Camaragibe (Figura 1). No que se refere a sua finalidade, as respostas apresentadas pelos entrevistados foram as mais pertinentes, ainda assim, nas duas localidades, cerca de $30 \%$ dos que o con heciam não sabiam para que servia. Em ambos os municípios, aproximadamen te $24 \%$ dos en trevistados afirmaram que este órgão tem por objetivo defender o consumidor. As demais res postas se referiam a temas pontuais, se bem que relacionados com aspectos econ ô mi cos que envolviam a proteção do consumidor. A prestação de informações e defesa dos direi to s, em geral, foram citadas por cerca de 7\% no Ca bo e 12,3\% em Camaragibe. Nos dois municípios, foi inex pressiva a proporção de entrevistados que relacionou o Procon com a saúde (Figura 7).

Apesar de muito conhecido, apenas cerca de $4 \%$ dos entrevistados, de ambas as localidades, tiveram ex periência com o Procon (Figura 2). Os motivos da procura foram vários e, geralmente, relacionados com problemas de ordem econômica. No que diz respei to às respostas às demandas, a maior parte dos entrevistados considerou positiva as respostas às suas queixas, $53 \%$ no Cabo e $72 \%$ em Camaragibe.

\section{Figura 6}

Distri buição percentual das res postas rela tivas à finalidade do Disque-Saúde. Ca bo de Santo Ago s tinho e Camaragibe, 2000.




Figura 7

Distri buição percentual das re spostas rel a tivas à finalidade do Procon . Ca bo de Santo Agostinho e Camaragibe, 2000.

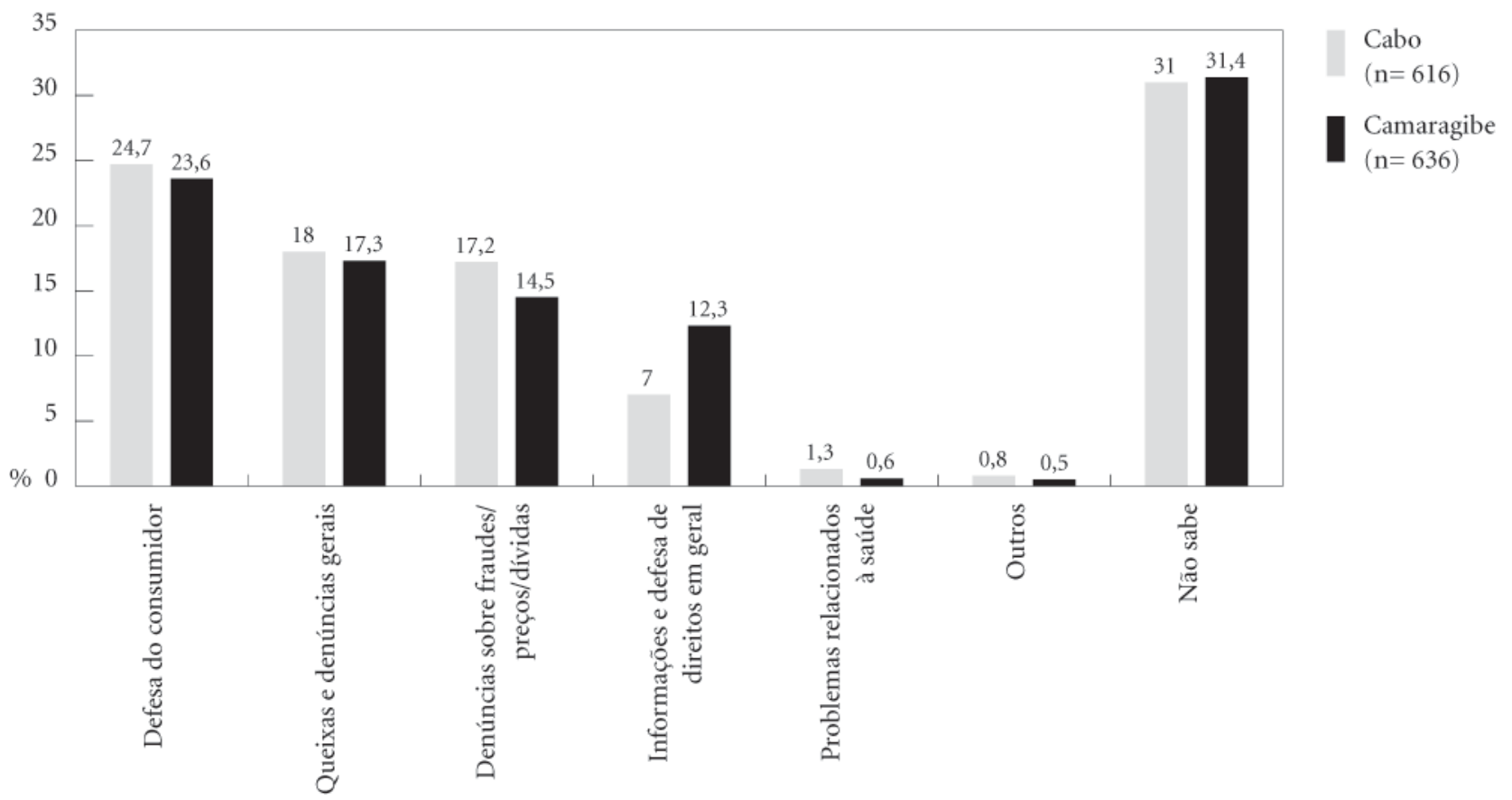

Uma menor parcela de usuários, porém, ainda significativa, con s i derou negativo o retorno do Procon, 31,3\% no Cabo e 10,3\% em Camaragibe. Os demais en trevistadbs esperavam resposta no mom en to da en trevista.

\section{Formas de participar na própria unidade de saúde}

Procurou-se iden tificar que outros mecanismos de participação os usuários conheciam nos serviços de saúde. Pa ra tanto, foi perguntado se existia, nas unidades de saúde que eles habitualmen te utilizavam, um local ou pessoa responsável para receber as qu eixas e su gestões. Em ambos os municípios cerca de $24 \%$ dos entrevistados responderam que existia. Porém, 22,8\% no Cabo e 29,7\% em Camaragibe afirma ram que não existia, e, aproximadamente, a metade dos entrevistados não sabia se existia ou não (52,9\% no Cabo e 45,8\% em Camaragibe). Entre aqueles que afirm a ramaexistência de umapessoa ou de um local para recepção de qu eixas e sugestões, a maioria $(74,0 \%$ no Ca bo ; $81,7 \%$ em Camaragi be) referiu um servi dor da unidade de saúde e um outro gru po (16,6\% no Cabo e $8,4 \%$ em Camaragibe) afirmou que exis tia uma caixa de qu eixas e sugestões. Tanto no Cabo, como em Camaragibe, 4,6\% dos entrevistados afirmaram que já haviam procurado o referido servidor. Em ambos os municípios, as principais razões da procura foram os problemas com a organização dos serviços e com a prestação da assistência à saúde. Con cernente às respostas relativas às queixas, no $\mathrm{Ca}$ bo, mais da metade considerou o resultado bom ou parcialmente bom, enquanto os dem a is disseram que não houve re s posta ou desconheciam os resultados. Es ses resultados ocorreram de forma inversa em Camaragibe.

Em relação ao sistema de saúde, de modo geral, perguntou-se a todos os entrevistados qual era a pessoa ou local que normalmente procuravam para prestação de queixas ou sugestões. Cerca de 35,5\% no Cabo e 47,7\% em Camaragibe indicaram algum trabalhador $\mathrm{da}$ unidade de saúde. Foram citadas outras instâncias, como, por exemplo, a prefeitu ra ou a sec retaria de saúde, por $16,4 \%$ dos usuários do Cabo e por $8,7 \%$ dos de Camaragibe. Um terço 
das pessoas respon deu que "não procuravam ninguém e não tinham a quem procurar", tanto no Cabo $(33,7 \%)$, como em Camaragibe $(28,6 \%)$. Ao serem perguntados se já haviam procurado o local ou pessoa mencionados, a proporção de usuários do Cabo (9.4\%) que re sponderam afirmativamen te foi significativamen te men or $(p<0,05)$ do que entre os de Camaragibe $(13,2 \%)$

\section{Discussão}

A amostra pode ser considerada repre sen ta tiva da população dos municípios estudados, devido a sua naturezaprobabilística, assim como os resultados, uma vez que a estrutu ra demográfica en con trada em ambas as populações é similar à da população geral, on de exis te uma concentração de pessoas nos estra tos mais jovens e uma maior proporção de mulheres.

Os dados socioecon ô micos são igualmen te compatíveis com os procedentes de outras fontes. In dicadores socioecon ô mi cos como escolaridade e ocupação revelam uma situação desfavorável nas duas comunidades, onde as proporções en contradas de analfabetismo foram superiores à taxa média de analfabetismo no Brasil, que atinge a cifra de $12,3 \%$, em maiore s de 10 anos (Ministéri da Saúde, 2002b). A situação mais desfavorá vel foi observada no Cabo. Pa rece existir, porém, uma tendência de melhoria em ambos os municípios, uma vez que se observa uma redução deste índice, que, durante a década de 80 , situava-se em torno de 30-40\% (Ação Educativa, 2000). Acompanhando a tendência nacional, as mulh eres apres entaram um perfil educacional discretamente melh or que o dos homens. Uma das possíveis explicações para esta diferenciação de gênero poderia ser a com binação da pobreza e do trabalho infantil, que, com mais freqüência, impelem o grupo de crianças e adolescen tes do sexo masculino a con tri buir com o su sten to familiar, levando-os, muitas vezes, a desempenhar atividades incompatíveis com o horário escolar (Inep, 2002).

As duas populações apresentam um perfil laboral precário, com uma taxa de desemprego $(10,5 \%)$ semelhante à registrada em nível naci onal $(9,8 \%)$. Refleti n do a realidade brasileira, a inserção das mulheres no mercado de trabalho é mais desfavorável e o trabalho manual não qualificado é a ocupação mais freqüen te deste grupo. Informações do Departamen to In tersindical de Estu dos Estatísticos corroboram estes resultados e indicam que, apesar do maior nível de instrução, em relação aos homens, as mulh eres não exercem funções compatíveis com a sua formação, ocupando, em maior percentual, postos mais precários, além de perceberem men or remu n eração (Dieese, 2000). Situação sem el han te a outros paises (Artazcoz et al., 2003).

$\mathrm{O}$ alto percentual de entrevistas realizadas com pen si onistas e aposentados parece coerente, pois é esperado que as pessoas que trabalham não estejam em casa nos hor á rios em que foram realizadas as entrevistas. Espera-se, igualmente, que os grupos de idade mais avançada padeçam maior número de doenças, considera n do que o cri t é rio de inclusão na amostra era ter utilizado os serviços de saúde nos últimos quatro meses. Também contribuiu para a elevação deste percentual o número de pessoas aposentadas com idade entre 43 e 48 anos. A maioria delas, trabalhadores pou co qualificados, que exerciam ativid ades "braçais", de sga stantes e espoliativas, iniciadas na idade em que deveriam estar na escola. A diferença nas proporções de homens e mulheres aposentados pode ser resultante da desigual forma de inserção das mulheres no merc ado laboral. Além da taxa superi or de ocupação registrada en tre os homens, este grupo ocupa também os postos de trabalho de melhor qualidade, os quais podem assegurar a relação de emprego formal. Desta forma, os hom ens têm maior garantia em termos de ben efícios sociais resultantes da "carteira assinad a”, den tre eles, a aposen tadoria .

De uma maneira geral, pode-se considerar que ainda é limitado o nível de informação da população estudada sobre os mecanismos institu ci onais de participação, qu er seja individual ou coletivo. Embora ocorra certa difusão da existência dos diferen tes mecanismos entre a população, uma proporção considerável dos en trevi s t ados afirmou de s conhecer os mecanismos institucionais diretos de participação. A gra nde maioria tinha con hecim en to apenas do Procon, o mecanismo institu cional indireto estudado. Do mesmo modo, em ambas as localidades, o objetivo de cada um dos mecanismos foi definido por ações pontuais ou expressões genéricas relacionadas com a atuação dos serviços de saúde, in dican doum conhecimen to limitado da população sobre os mecanismos de participação social. As causas des te baixo nível de informação são, provavelmente, múltiplas. 
A existência do CMS era con hecida por qu ase met ade da população estudada em Camaragibe e numa proporção um pouco menor no Cabo, em bora apenas uma parte desses entrevistados soubesse explicar a sua finalidade. Mu ito men or ainda era a informação da população sobre as conferências de saúde. Estes são os dois principais mecanismos institucionais de participação social em saúde, estabelecidos com caráter de obrigatoriedade no marco legal federal. Sua existência e suas funções deveriam s er mais bem con h ecidas pela população, uma vez que são os mecanismos em que deveria ter lu gar a con sulta ampliada à sociedade (Ba rros, 1994), sen do, portanto, essenciais na dem ocratização dos serviços de saúde. Por um, l ado este resultado indica uma difusão limitada das informações sobre a atuação dos CMS e conferências. Por outro lado, leva a questionar o papel dos conselhei ros comunitários e dos participantes nas conferências, quan to a sua capaci$\mathrm{d}$ ade de reco $\mathrm{lh}$ er e ex pressar os interesses da socied ade que repre sentam (Pessoto et al., 2001; Wendhausen e Caponi, 2002; Labra, 2002). Os conselh ei ros são os receptores das capacitações s obre os CMS e deveriam constituir-se em elos en tre a comunidade e os serviços de saúde. Um elemen to que pode estar influin doé a existência de uma ampla tradição de com portamentos a utoritários, em todos os níveis de governo, que levam à con cen tração de informações e tomada de decisões na cúpula do poder, s em que exista, em verdade, um intercâmbio de informações e opiniões com a população, sen do re stringida a participação dos conselheiros comunitá rios (Valla, 1998b; Si lva \& Labra, 2001).

O disque-saúde e o Procon, dois mecanismos institucionais de participação individual, foram os mais con hecidos pelos en trevistados. Is to poderia ser resultante das informações que aparecem com certa freqüência sobre estes mecanismos nos diversos meios de comunicação de massa (o número do disque-saúde, por exemplo, figura nos jornais, na seção de utilidade pública, junto com outros números de tel efone de urgências), visto que também se identifica um alcance limitado do conhecimen to em relação às suas características como mecanismo de participação em saúde. O disque-saúde funci onapara a população en trevistada, principalmente, como um veículo de informações ou facilitador do atendimento. O Procon, a pesar de o mais conhecido é relacionado apenas como um serviço de caráter estritamente econômico e não por sua possível relação com os serviços de saúde. Por outro lado, é import a n te destacar que se o Procon é con h eci do por seu papel econômico, isso se deve à rel evância naci onal conquistada com a difusão de informações acerca de sua atuação em causas rel acionadas, principalmente, com o sistema financei ro habitacional. Foi a atuação do órgão, dan do re sposta aos probl emas apre sen tados, o que con tribuiu para que o serviço fosse divulgado na mídia e ganhasse a confiança e apoio da população (Fundação Procon São Paulo, 2001).

A apreciável proporção de entrevistados (42\%) que conhecia as caixas de qu eixas e sugestões parece que está influenciada pela existência destemecanismo em diversificados estabel ecimentos, freqü en tados habi tualmen te pela população, como por exemplo, os superm erc ados. Fato corroborado pela quantidade de respostas afirmativas em ambos os municípios, a pesar de terem disponível este mecanismo de forma restrita nos postos e centros de saúde e também pelas res postas que indicavam outros motivos para havê-las utilizado, além dos relacionados com a saúde. É importante destacar, porém, o potencial destemecanismo, vis to que uma boa proporção dos entrevistados mostrava uma opinião positiva acerca do processamento das queixas, mas sem esquecer que para sua efetivação deveria estar inserido num sistema que permitisse aos serviços dar uma resposta (Calnan et al., 1998).

Em Camaragibe, o nível de informação sobre os mecanismos de participação em saúde foi consisten tem en te melhor do que em Cabo. As causas desta melhor difusão do conhecimento em Camaragibe necessitam de maior análise. Poderia ser resultante de uma possível di ferença da atuação dos mecanismos de acordo com a forma de gestão dos serviços de saúde; de um processo de municipalização mais an tigo (por exemplo, o CMS iniciou atividades em Camaragibe em 1992 e no Cabo em 1997); ou ainda de um melhor acesso à informação, por se tratar de uma população predomin a n temen te urbana, mais próxima da capital.

Dentre os mecanismos institucionais de participação, as mais altas taxas de utilização correspondem ao Procon, às caixas de queixas e su gestões e não se referem ao âmbito da saúde. Estas cifras são inferi ores àqu elas en con tradas no estudo realizado na Colômbia, onde o Serviço de Atenção ao Usuário dos serviços de saúde apresen tou uma taxa de utilização de 8,6\% (Mosquera et al., 2001). O percen tual de participação, porém, é mais próximo ao estu do 
da Colômbia (aproximadamente 11\%), quando se consideram as queixas diretas ao pessoal de saúde, nos cen tros ou em outras instâncias do sistema de saúde. A carência de parâmetros de comparação leva ao questionamen to de qual seria o nível de utilização dos mecanismos de participação que poderia ser con si derado aceitá vel ou desejável, leva n do-se em con si deração os obj etivos alcançáveis por meio de cada mec anismo. Nesse sentido, o estu do proporciona uma base de referência para futu ras comparações.

A participação indivi dual ou coletiva sempre pressupõe custos, tanto de tempo, como psicológicos. Nesse sentido os individuos podem decidir não participar, só atua rem se perceberem que têm a capacidade de influenciar nos resultados, que a sua pre sença é imprescindível, e que con seguirão ben efícios (Klein, 1984). A utilização dos mecanismos de participação institucionais pode ser uma opção inadequada se a população não acredita que possa levar a mudanças (Valla, 1998b). A freqüência da qu eixa oral direta poderia indicar a necessidade de se implantarem mecanismos por meio dos quais seja possívd uma interação direta usuário-pessoal de saúde, como, por exemplo, um serviço de atenção ao usuário, em que se possa obter uma resposta ao problema (Vázquez et al., 2003). A proporção não desprezível dos entrevistados que afirmou "não procurar ninguém" nos serviços de saúde, poderia indicar uma falta de confiança nos resultados, uma vez que, em ambos os municípios, registrou-se uma proporção con si der á vel de pessoas que ac redita que os serviços não tomam nenhuma providência com as demandas apresentadas por intermédio das caixas de qu eixas e sugestões. An alisandoa ex periência do Procon, é plausível esperar que a informação e confiança da população sobre estes mecanismos de participação melhorarão na medida em que os re sult ados de suas atuações sejam também conhecidos e respondam às necessidades da população.

\section{Con clusão}

Segundo Stone (1992), um grande problema na promoção da participação no contex to da atenção primária de saúde é que se trata de uma política imposta desde os níveis cen trais e que enfrenta oposição dos distintos níveis do sistema, sen do, em gran de parte percebi do, como um imposição ex terna. No Brasil, as políticas de participação social são o resultado de anos de luta e pressões pela democratização do Estadb (Pessoto et al., 2001; Silva \& Labra, 2001). Estas políticas levaram a mudanças organizacionais com a criação de diversificadas portas de entrada indivi duais e coletivas para a participação da população no sistema de saúde. Os resultados deste estudo evidenciam, porém, que o desafio conti nua sendo melh orar a forma de pôr em prática as con quistas estabelecidas no plano legal (Bosi \&Af fonso, 1998).

Apenas uma parte da população con h ece a existência dos mecanismos institucionais de participação coletivos e individuais e a informação se mantém em níveis superficiais. Do mesmo modo, só uma pequena proporção da população utiliza os mecanismos institucion a is , situando a população estudada no patamar da in formação (Arstein, 1969), ainda lon ge do almejado con trole social. Estes resultados apontam a debilidade das estratégias de divulgação da existência dos mecanismos e de como funcionam os resultados de suas atuações e da informação de como participar. O individuo e a coletividade devem dispor da suficien te informação sobre a atuação dos serviços de saúde para determinar se, como, quando e com qu em deve atuar. A informação é fundamental para o reconhecimen to dos mecanismos como opção para a atuação jun to aos serviços. Is to implica proporcionar um con ju $\mathrm{n}$ to de ações que requerem a divulgação destes mecanismos, como também a apre sentação de respostas às necessidades da população que reforçarão o conheci$m$ en to e a confiança nestes mecanismos. 
Colaboradores

Todos os autores, exceto MRF Si lva e APC Perei ra, participaram no des enho da pesquisa. Exceto MRF Silva, os demais colaboraram no trabalho de campo para a coleta dos dados. ML Vázquez e MRF Silva levaram a cabo a pe squisa bibliográfica, a análise dos dados e lideraram a redação do arti go, que recebeu as con tribuções dos autoresrestan tes. ML V á z quez fez a coordenação e supervisão geral do trabalho.
Agradecimentos

O estudo foi realizadocomo colaboração en tre o Institute for Health Sector Development (Reino Unido), Instituto Materno-Infantil de Pernambu co (IMIP), Univers i$\mathrm{d}$ ade Federal de Pernambuco (Brasil), Escuela Andaluza de Salud Pública (España), Un iversidad del Valle (Colombia), com o apoio de Consorci Hospitalari de Catalu nya e as Sec retarias de Saúde dos Municípios de Cabo de Santo Agostinho e de Camagaribe. Agradecemos às pes s oas en trevis tadasem ambos osmunicípios, que aceit a ram compartilhar com ospesquisa dores suas opiniões e con ceitos. À Comissão Européia Di rectora te General XII eMinistério da Sa ú de do Brasil, cujo apoio financeiro foi imprescindível para a realização do estu do.

\section{Referências bibliográficas}

Abrantes-Pêgo R 1999. Participación social en salud: Un estudio de caso en Brasil. Salud Publica Mexicana 41(6):466-474.

Ação Edu ca tiva 2000. InformAÇÃO em red e. Ação edu cativa - Assessoria, Pesquisa e Informação. Disponível em $<$ http://www.aca oeducativa.org/IR25.pdf $>$. Ace sso em 20 de junho de 2002.

Araújo JL Jr. 1997. At tempts to decen tralise in recent Brazilian health policy: issues and problems, 1988-1994. In ternational Jou rnal of Health Services 27(1): 109-124.

Ars tein SR 1969. A lad der of participation. American Institu te of PlannersJournal 35:216-224.

Artazcoz L, Cortès I, Ben ach J \& Ben avi des FG 2003. Les desigualtats en salut laboral, pp. 253-282. In C Borrell \& J Ben ach (coords.). Les desigualtats en la salut a Catalunya. Editorial Med iterrània, Ba rcel on a .

Barros E 1994. O con trole social e o processo de descentralização dos serviços de saúde, pp. 29-37. In In centivo à participação popular e controle social no SUS (Tex tos técnicos paracon selh eiros de saúde ). Ministério da Sa ú de, Brasília.

Bennett S 1998. Reforming State capacity: the demands of health sector reform in developing countries. Forum on Health Sector Reform. Lon don School of Hygiene and Tropical Medicine, 21-24 April 1998 (mimeo).

Bosi MLM \& Af fonso KC 1998. Gdadania, participação e saúde: com a palavra, o usuário da rede pública de s erviços. Cadernos de Saúde Pública 14(2):355-365.

Brasil 1991. Mnistério da Saúde. Norma Operacional Básica. Inamps no 1/91. Resolução no 273/91. Diário Oficial da União Brasília, p. 14216-9, 18 de julho. Seção I.
Brasil 1992. Ministério da Saúde. Norma Operacional Básica - SUS no 2/92, Portaria no 234/92. Diário Oficial da União. Brasília. Seção I.

Brasil 1996. Ministério da Saúde. Norma Operacional Básica do Sistema Único de Saúde. (NOB-SUS/96). Portaria no 2203. Diário Oficial da União. Brasília. 6 de novem bro. Seção I.

Calnan M, Halik J \& Sa bbat J 1998. Citi zen participation and patient choice in health reform, pp. 325-338. In RB Saltman, J Figueras \& C Sakellarides. Critical challen ges for health care refo rm in Eu rope. Open Un iversity Press, Buckinghan.

Celedón C \& Noé M 2000. Reformas del sector de la sa lud y participación social. Revista Panamericana de Salud Pública/Pan Am erican Journal of Public Health 8(1/2):99-104.

Cohn A 2000. Cidadania e fo rmasderespon sabilização do pod er públicoe do setor privado pelo aces so, equidade, qualidade e humanização na atenção à saúde. Disponível em <http:www.saude.gov.br/11cns/index_11 cns.htm >. Acesso em 4 de junho de 2001.

Cotta RMM, Suárez-Varela MM, Claver SF, Cotta-Filho JS \& Llopis-González A 2000. Participación social y sistemas sanitarios en Brasil Vs España. Centro de Salud 8: 450-455.

Côrtes SMV 2002. Balanço de experiências de controle social, para além dos co n selhos e conferências no Si s tema Un nicode Saúde brasileiro: Construindo a possibilidade de participação dos usuários. Disponível em $<\mathrm{http}$ : www.saude.gov.br/11cns/cns_balanco.htm $>$. Acesso em 4 de junho de 2001.

Dias JCP 1998a. Problemas e possibilidades de partici- 
pação comunitária no controle das grandes en demias. Cadernos de Saúde Pública 14(supl. b2):19-37.

Dias RB 1998b. "Eu? Eu estou aí, compon do o mundo". Uma ex periência de con trole de endemia, pesquisa e participação popular vivida em Cansanção, Minas Gerais, Brasil. Cadernos de Saúde Pública 14(supl. 2):149-157

Di eese - Departamen to In tersindical de Estatística e Estu dos Socioeconômicos. 8 de março de 2000 - Dia Internacional da Mulher. Disponível em <http:// www.dieese.org.br/esp/mulher.htm >.

Fundação Procon São Paulo 2001. B reve histórico da proteção ao consumidor. Disponível em $<\mathrm{http}$ ://www. procon.sp. gov.br/institucionalhistorico.htm $>$. Ace sso em 18 de março de 2002.

González RE 1996. Ma nual sobre participación y organización pa ra la gestión local. Ediciones Foro Nacional Por Colombia, Cali.

GTCOM/Abras so 2002. Políticas de comunicação para o SUS: Co municação e controle Social. Disponível em $<$ http://www.abrasco. org.br $>$. Acesso em 18 de março de 2002.

Insti tuto Nacional de Estudos e Pesquisas Educacionais (Inep) 2002. Mulheres ganham dos homens em nivel de Instrução. Disponível em <http://www.inep.gov. br/imprensa/noticias/outras/news00_25.htm >. Acesso em 20 de junho de 2002.

Kl ein R 1984. Thepolitics of participation, pp. 17-32. In $\mathrm{R}$ Maxwell \& $\mathrm{N}$ Weaver. Public participation in health. King Edward's Fund, Lon don.

Labra ML 2002. Capital social y con sejos de salud en Brasil. ¿Un círculo virtuoso? Cadernos de Saúde Pública 18(supl.):47-55.

Mnistério daSaúde 2002a. 11a Conferência Nacional de Saúde. Relatório Final. Disponível em $<\mathrm{http}: / / \mathrm{www}$. Datasus11con feren cia/rel a torios/proposiçoes.htm.g ov.br/cns/>. Acesso em 8 de ja n ei ro de 2002

Mnistério da Saúde. 2002b. Info rmações demográficas e socioeconômicas. Dis ponível em <http://www.datasus.gov.br/>. Acesso em 20 de junho de 2002.

Mosquera M, Zapata Y, Lee K \& Varela A 2001. Strengthening user participation through health sector reform in Colombia: a study of institutional change and social representation. Health Policy and Planning 16(2):52-60.

OMS Organización Mundial de la Salud 1978. Primary Health Care. Health for All series no. 1. World Health Organisation, Geneva.

OMS Organización Mundial de la Salud 1986. Ottawa Charter for Health Pro motion. An international conference on health promotion. WHO/Health and Welfare Canada/Canadian Public Health Association. Ottawa, Canada.

OMS Organización Mundial de la Sa lud 1988. The Adelaide Recommendations, Healthy Pu blic Policy. WHO/ Eu rope, Copen h a gen.
OMS Organización Mundial de la Salud 1989. Call for Action. Promoting Health in Developing Countries. WHO, Geneva.

OMS Orga nización Mundial de la Sa lud 1995. Suppo rtive environments for health statement. WHO/HPR/HEP/ 95.3. Geneva.

OMS Organizadón Mundial de la Salud 1996. Eu ropean Health Care Reforms. Analysis of Current Strategies. Summary. Copenhagen: WHO Regional Office for Europe.

Pedrosa JIS 1997. A construção do significado de controle s ocial com con sel hei ros de saúde no Estado do Piauí, Brasil. Cadernos de Saúde Pública 13(4):741-748.

Pessoto UC, Nascimento PRD \& Heimann LS 2001. A gestão semiplema e a participação popular na administração da saúde. Cadernos de Saúde Pública 17(1):8997.

Silva IFD \& Labra ME 2001. As instâncias colegi adas do SUS no Estado do Rio de Jan ei ro e o processo decisório. Cadernos de Saúde Pública 17:161-170.

S tone L 1992. Gultu ral influences in community participation in health. Social Science \& Medicine 35(4): 409-417.

Valla VV 1998a. Participação popular e en demias: uma nova conjuntura. Cadernos de Saúde Pública 14(supl. 2):4-5.

Valla VV 1998b. Sobre participação popular: uma questão de pers pectiva. Cademos de Saúde Pública 14(supl. 2):7-18.

Walt G \& Gilson L 1994. Reforming the health sector in developing countries: the central role of policy a $\mathrm{n}$ a lysis. Health Policy andPlanning 9(4):353-370.

Vázquez ML, Siqueira E, Kruse I \& da Si lva A 2002a. Los procesos de reforma y la participación social en salud en Am é rica Latina. Ga ceta Sanitaria 16(1):30-38.

Vázquez ML, da Si lva MRF, Siqueira E, Perei ra AP, Diniz ADS, Lei te I \& Kru ze I 2002b. Visión de los diferen tes agentes sociales sobre la participación social en el sistema de salud en el Nordeste de Brasil. Una aprox im ación cualitativa. Revista Es pa ñ ola de Salud Pública 76(5):585-594.

Vázquez ML, da Silva MRF, Siqueira E, Kruze I, Diniz ADS, Leite I \& Perei ra AP 2003. Pa rticipação social nos serviços de saúde: concepções dos usuá rios e líderes comunitários em dois municípios do Nordeste doBrasil. Cademos de Saúde Pública 19(2):579-591.

Wendhausen A \& Caponi S 2002. O diálogo e a participação em um conselho de saúde em SantaCatarina, Brasil. Cadernos de Saúde Pública 18(6):1621-1628.

Woelk GB 1992. Gultural and structural influ en ces in the creation and participation in com mu nity health programmes. Social Scien ce and Med icine 35(4):419-424.

Zakus D \& Lys ack C 1998. Revisiting com mu nity participation. Health Policy and Planning 13(1):1-12.

Artigo apresentado em 15/01/2004

Aprovado em 21/07/2004

Versão final apresentada em 17/08/2004 